\title{
UN NUEVO ALGORITMO PARA LA CARTOGRAFÍA DE ÁREAS QUEMADAS A PARTIR DE IMÁGENES AWIFS Y MODIS
}

\author{
F. GONZÁLEZ-ALONSO(1), S. MERINO DE MIGUEL ${ }^{(2)}$ \\ y J. M. CUEVAS GOZALO(1)
}

(1) Laboratorio de Teledetección (CIFOR - INIA) Ctra. A Coruña km 7,5, Madrid 28040

(2) EUIT Forestal (Universidad Politécnica de Madrid) Ciudad Universitaria s/n, Madrid 28040

Correo electrónico de contacto: alonso@inia.es

\begin{abstract}
RESUMEN. El presente trabajo muestra un nuevo algoritmo para la producción de mapas de área quemada que sinérgicamente combina información de tres partes diferentes del espectro (infrarrojo cercano, de onda corta y térmico). Dicho algoritmo está basado en el uso de una variante del índice BAIM (Burned Area Index) e información tipo "hotspot". En particular, se ha utilizado una imagen AWiFS (Advanced Wide Field Sensor) tomada el 21 de agosto de 2006, anomalías térmicas captadas por MODIS (MODerate resolution Imaging Spectrometer) durante los primeros 20 días de agosto, así como otros datos auxiliares y cartografía. La metodología desarrollada fue testada en Galicia (España), donde cientos de incendios forestales ocurrieron durante los primeros días de agosto de 2006. Los mapas y resultados alcanzados fueron validados utilizando las estadísticas del Ministerio de Medio Ambiente y el Servicio Forestal Gallego. Los resultados alcanzados muestran que esta metodología puede ser de gran interés a escalas regionales y nacionales, ya que ha demostrado ser rápida, precisa y económica.
\end{abstract}

ABSTRACT. This paper presents a new algorithm that synergistically combines data from three different parts of the spectrum (near-, shortwave- and thermalinfrared) to produce reliable burned area maps. It is based on the use of a variation of the BAIM (Burned Area Index) together with hotspot information. In particular, the study presented herein uses an image from the AWiFS (Advanced Wide Field Sensor) sensor dated on the 21 st of August 2006, MODIS (MODerate resolution Imaging Spectrometer) hotspot detected during the first twenty days of August as well as ancillary maps and information. Developed methodology was tested in Galicia (North-West Spain) where hundreds of forest fires occurred 
during the first twenty days of August 2006. Burned area maps and results were validated using fire statistics from both the Spanish Ministry of Environment and the Galician Forest Service. Reached results showed that this method would be of great interest at regional to national scales, since it was proved to be quick, accurate and cost-effective.

Palabras clave: BAI, BAIM, hotspot, incendios forestales, Cuenca Mediterránea. Key words: BAI, BAIM, hotspot, forest fires, Mediterranean basin.

Enviado el 20 de junio de 2007 Aceptado el 25 de septiembre de 2007

\section{Introducción}

Los incendios forestales son uno de los problemas medioambientales y de seguridad más importantes de los países mediterráneos del sur de Europa. En esta área geográfica, los veranos son calurosos y secos lo que, unido al incremento de combustibles por abandono del medio rural, está llevando a un notable incremento del área afectada por incendios forestales. Los incendios forestales causan importantes pérdidas económicas, sociales y medioambientales. Además de destruir notablemente el paisaje y los ecosistemas, los incendios forestales constituyen una importante fuente del principal gas de efecto invernadero (dióxido de carbono), lo que repercute en el calentamiento de la atmósfera y en la alteración del sistema climático global.

La utilización de métodos tradicionales para la cartografía del área afectada por incendios forestales es cara y lenta. La evaluación y cartografía de dichas áreas mediante técnicas de teledetección presenta en cambio notables ventajas, por lo que se están utilizando cada vez más para abordar este problema. Las estimaciones realizadas mediante técnicas de teledetección son rápidas, fiables y económicamente viables, lo que permite además el establecimiento de sistemas de respuesta rápida. Además, estos mapas pueden combinarse con información sobre la pendiente o el tipo de suelo con el objetivo de establecer áreas de intervención prioritaria y planificar adecuadamente los trabajos de restauración (González-Alonso et al., 2007).

Entre los métodos que existen para cartografiar superficies quemadas mediante teledetección, destacan los siguientes (Koustias et al., 1999): (i) establecimiento de umbrales a partir de bandas originales o índices, (ii) clasificación supervisada de las bandas originales o índices, (iii) clasificación no-supervisada, (iv) análisis multivariante de las bandas originales, (v) análisis de mezclas espectrales, (vi) análisis de series temporales, etc. Entre todos ellos se ha elegido una variante del establecimiento de umbrales a partir de índices.

El objetivo del trabajo que se presenta consiste en estimar y cartografiar las áreas afectadas por los incendios forestales ocurridos en Galicia durante la primera quincena del mes de agosto de 2006. Para llevar a cabo dicho trabajo se ha utilizado infor- 
mación procedente del sensor Resourcesat-1 - AWiFS (Advanced Wide Field Sensor) y del sensor TERRA/AQUA - MODIS (Moderate resolution Imaging Spectrometer) tal y como se explica en la sección "Material". En particular, la imagen AWiFS fue utilizada para obtener una variante del índice BAI (Burned Area Index, Martín et al., 2005) que sirvió a su vez para obtener un mapa de quemado - no-quemado. Los datos MODIS, que consistieron en series temporales de anomalías térmicas o "hotspots", fueron utilizados en dos tareas: (i) establecer el umbral de BAI que separaba quemado de no-quemado y (ii) eliminar errores de comisión, tal y como se explica en la sección "Métodos".

\section{Material}

Para llevar a cabo el presente trabajo se utilizaron tres fuentes de información: (i) una imagen de satélite post-incendio, (ii) una serie temporal de anomalías térmicas o hotspots y (iii) una serie de mapas y datos auxiliares.

Se utilizó una única imagen de satélite del sensor AWiFS (a bordo del satélite indio Resourcesat-1) captada el 21 de agosto de 2006 (Figura 1). También se utilizaron datos MODIS hotspot correspondientes a los 20 primeros días de agosto de 2006 (Figura 1). "AWiFS es un sensor único, capaz de proporcionar datos a $56 \mathrm{~m}$. de resolución espacial, con cinco días de resolución temporal" (Kulkarni et al., 2006) y en cuatro bandas espectrales (Tabla 1). MODIS es un sensor de alta resolución temporal que se encuentra a bordo de los satélites TERRA y AQUA de la NASA, captando información en más de 30 canales de resolución espacial variable, dos de los cuales (de $1.000 \mathrm{~m}$. de tamaño de píxel) son canales térmicos utilizados en la producción de datos hotspot.

Los datos de anomalías térmicas de MODIS, tal y como fueron facilitados por el MODIS Land Team de la NASA, consistieron en una serie de archivos de texto (uno por día), cada uno de los cuales presentaba un registro por cada anomalía detectada, para la que daba la siguiente información: localización (longitud y latitud), fecha, hora, nivel de confianza de la estimación y satélite que había realizado la detección (TERRA o AQUA). Para el área geográfica de Galicia (alrededor de tres millones de hectáreas), y correspondiendo a los 20 primeros días de Agosto, fueron detectados 3.340 hotspot. 


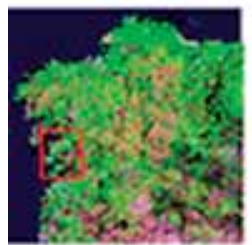

(a)

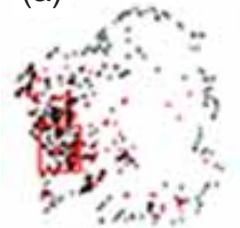

(b)

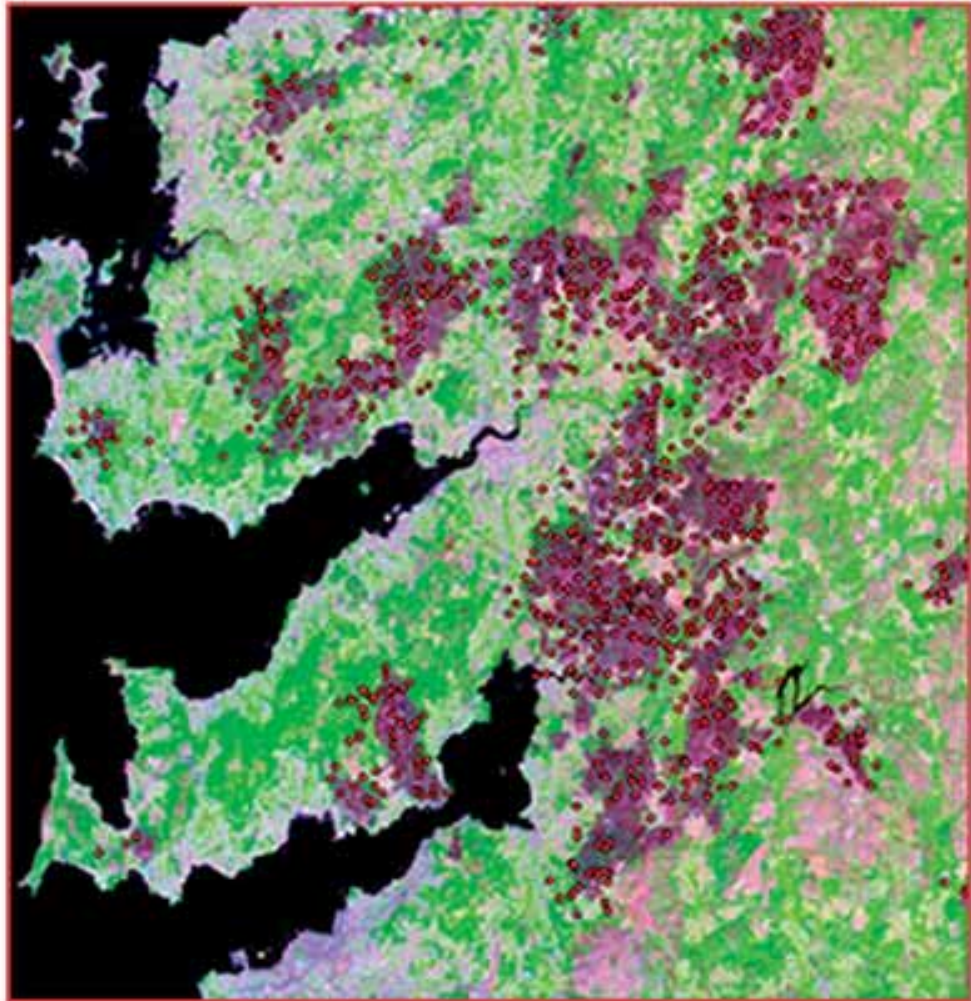

(c)

Figura 1. (a) imagen AWiFS post-incendio (21 de agosto de 2006, composición RGB: 543), (b) anomalías térmicas MODIS (del 1 al 20 de agosto de 2006), (c) detalle del área de estudio en el que se observa la coincidencia de machas quemadas y anomalías térmicas

Tabla 1. Características del sensor AWiFS (NIR: infrarrojo cercano; SWIR: infrarrojo de onda corta)

\begin{tabular}{|l|c|c|c|c|}
\hline Características AWiFS & $\begin{array}{c}\text { B2 } \\
\text { (verde) }\end{array}$ & $\begin{array}{c}\text { B3 } \\
\text { (rojo) }\end{array}$ & $\begin{array}{c}\text { B4 } \\
\text { (NIR) }\end{array}$ & $\begin{array}{c}\text { B5 } \\
\text { (SWIR) }\end{array}$ \\
\hline Resolución espacial & $56 \mathrm{~m}$. & $56 \mathrm{~m}$. & $56 \mathrm{~m}$. & $56 \mathrm{~m}$. \\
Ancho de barrido & $740 \mathrm{~km}$. & $740 \mathrm{~km}$. & $740 \mathrm{~km}$. & $740 \mathrm{~km}$. \\
Resolución temporal (días) & $2-5$ & $2-5$ & $2-5$ & $2-5$ \\
Resolución espectral (nm.) & $520-590$ & $620-680$ & $770-860$ & $1.550-1.700$ \\
\hline
\end{tabular}

Los mapas y datos auxiliares utilizados consistieron en: (i) un modelo digital de elevaciones que se utilizó en el pre-procesado de la imagen AWiFS y (ii) una cobertura CORINE Land Cover 2000 (CLC2000) que se utilizó para construir una máscara de 'quemable - no-quemable'. 


\section{Métodos}

\subsection{Pre-procesado de la imagen AWiFS}

Con el fin de obtener una imagen adecuada al procesado posterior, se realizaron correcciones radiométricas y geométricas sobre la imagen AWiFS original. Las correcciones radiométricas consistieron en realizar la calibración del sensor (conversión de los números digitales en valores de radiancia) para a continuación estimar reflectancias. No se realizaron correcciones atmosféricas.

Las imágenes de reflectancia 'top-of-atmosphere' resultantes fueron entonces corregidas geométricamente (orto-rectificadas) para lo que se utilizó un modelo digital de elevaciones (de $25 \mathrm{~m}$. de resolución especial) y los ficheros 'RPC' (archivos de calibración geométrica). El pre-procesado de la imagen se realizó utilizando el software ENVI 4.2.

\subsection{Estimación de la superficie afectada}

La metodología empleada en la cartografía de las áreas quemadas consta de los siguientes pasos: (i) cálculo del índice BAI, (ii) determinación del umbral de BAI que separara área quemada de no quemada de forma más precisa, (iii) aplicación de una máscara derivada del CLC2000 y (iv) análisis de coincidencia con hotspot para la producción final del mapa de áreas quemadas. En el tratamiento de la imagen y de la información se utilizaron ENVI 4.2 y ArcView 3.2.

El índice de área quemada (BAI) se diseñó específicamente para la discriminación de áreas afectadas por el fuego. "Este índice se calcula a partir de la distancia espectral de cada píxel a un valor de referencia, valor al cual convergen las áreas recientemente afectadas por el fuego" (Chuvieco et al., 2002). Dicho índice tiene la siguiente expresión:

$$
\mathrm{BAI}=\frac{1}{\left(p c_{n i r}-\rho_{\text {nir }}\right)^{2}+\left(p c_{\text {swir }}-\rho_{\text {swir }}\right)^{2}}
$$

Siendo: $\quad p c_{n i r}=$ reflectancia de referencia en el infrarrojo cercano, $\rho_{n i r}=$ reflectancia píxel en el infrarrojo cercano, $p c_{\text {swir }}=$ reflectancia de referencia en el infrarrojo de onda corta, $\rho_{\text {swir }}=$ reflectancia píxel en el infrarrojo de onda corta.

Las reflectancias de referencia se obtuvieron mediante análisis estadístico de píxeles quemados de las bandas NIR y SWIR de la imagen post-fuego AWiFS. Para ello, se digitalizaron más de 150 áreas de entrenamiento utilizando tanto combinaciones RGB de las bandas originales como los índices espectrales NDVI (Normalized Difference Vegetation Index) y NBR (Normalized Burn Ratio Index). En particular, se asignó como reflectancia de referencia en el NIR (Úc ${ }_{n i r}$ ) el percentil del cinco por ciento de la reflectancia en el NIR y, como reflectancia de referencia en el SWIR (Úc $c_{\text {swir }}$ ), el percentil del 95 por ciento de la reflectancia en el SWIR. 
El establecimiento del umbral de BAI que separa área quemada de no quemada, de forma más precisa, estuvo basado en la búsqueda de la mejor correlación entre 'área quemada' y 'número de hotspot de MODIS' para distintos tamaños de malla (de 1, 2, 3 y 4 $\mathrm{km}$. de lado). El 'área quemada' se estableció convirtiendo a vectorial la imagen binaria resultante de aplicar cierto umbral de BAI y conservando únicamente aquellos polígonos que contuvieran, al menos, un hotspot. Los valores de BAI utilizados para dicho análisis fueron: 80, 83, 85, 90, 95 y 100. Los mejores resultados, en términos de coeficiente de determinación, se obtuvieron para un umbral BAI de 90, independientemente del paso de malla utilizado (Tabla 2). La aplicación de dicho umbral sobre la imagen BAI original produjo un mapa de 'quemado - no-quemado'.

Tabla 2. Coeficientes de determinación entre valores de 'área quemada' y 'número de hotspot' para distintos umbrales BAI y distintos pasos de malla

\begin{tabular}{|c|c|c|c|c|}
\hline BAI & $\mathrm{r}^{2}-1 \mathrm{x} 1 \mathrm{~km}$ & $\mathrm{r}^{2}-2 \times 2 \mathrm{~km}$ & $\mathrm{r}^{2}-3 \times 3 \mathrm{~km}$ & $\mathrm{r}^{2}-4 \times 4 \mathrm{~km}$ \\
\hline 80 & $41,16 \%$ & $59,63 \%$ & $66,62 \%$ & $70,22 \%$ \\
83 & $43,38 \%$ & $63,25 \%$ & $71,04 \%$ & $74,81 \%$ \\
85 & $44,85 \%$ & $65,69 \%$ & $74,02 \%$ & $78,63 \%$ \\
90 & $45,80 \%$ & $67,71 \%$ & $76,58 \%$ & $81,33 \%$ \\
95 & $45,07 \%$ & $67,02 \%$ & $76,18 \%$ & $80,98 \%$ \\
100 & $44,29 \%$ & $66,37 \%$ & $75,24 \%$ & $80,61 \%$ \\
\hline
\end{tabular}

El mapa ‘quemado - no-quemado' resultante se mejoró mediante aplicación de una máscara derivada del CLC2000. Es evidente que una buena cartografía de áreas quemadas no puede incluir láminas de agua, zonas urbanas o zonas sin vegetación, para lo que se aplicó dicha máscara. La imagen 'quemado - no-quemado' mejorada se convirtió a formato vectorial (capa de polígonos).

La capa de polígonos resultante del paso anterior se superpuso a una composición RGB de la imagen AWiFS con el objetivo de realizar un análisis visual de posibles errores de omisión y comisión. Galicia es un extenso territorio en el que, en su alrededor de 3 millones de hectáreas, podemos encontrar ecosistemas que van desde los asociados al clima Atlántico hasta los más Mediterráneos. Esta variabilidad espacial dio lugar a la aparición de una 'excelente delineación' de las manchas quemadas cercanas a la costa oeste, pero una 'fuerte aparición de errores de comisión' en las zonas del sureste (Figura 2). Sin embargo, y tal y como se observa en la Figura 1, la mayor parte de las manchas quemadas de la escena AWiFS presenta además un importante número de hotspot en su interior. Esta circunstancia motivó el último paso en la producción de la cartografía de áreas afectadas: la utilización de la localización de hotspot para la eliminación de los posibles errores de comisión así como la eliminación de polígonos de muy pequeño tamaño (Figura 3). Un último análisis visual sirvió para recuperar aquellos polígonos que, apareciendo claramente afectados por el fuego, habían sido descartados por no contener ningún hotspot. Esta última operación dio lugar al mapa final de áreas afectadas por el fuego (Figura 4). 


\section{Resultados}

El mapa final de área afectada por el fuego (Figura 4) corresponde a toda la superficie quemada en Galicia durante la campaña 2006 hasta el 21 de agosto. El área total se ha cifrado en 93.261 ha., más del tres por ciento del territorio gallego. Aunque no se realizó validación mediante datos externos (datos de campo, perímetros GPS, etc.), un análisis visual exhaustivo permite concluir que el resultado alcanzado fue bastante satisfactorio.

También conviene recordar que la cifra alcanzada en el presente trabajo de investigación resulta bastante acorde con las ofrecidas por los organismos oficiales. En particular, el Ministerio de Medio Ambiente (Ministerio de Medio Ambiente, 2006) cifra en 92.941 ha. la superficie afectada en Galicia hasta el 1 de octubre, mientras que para la Xunta de Galicia (Xunta de Galicia, 2007) el área afectada para todo el año 2006 es de 93.887 ha.

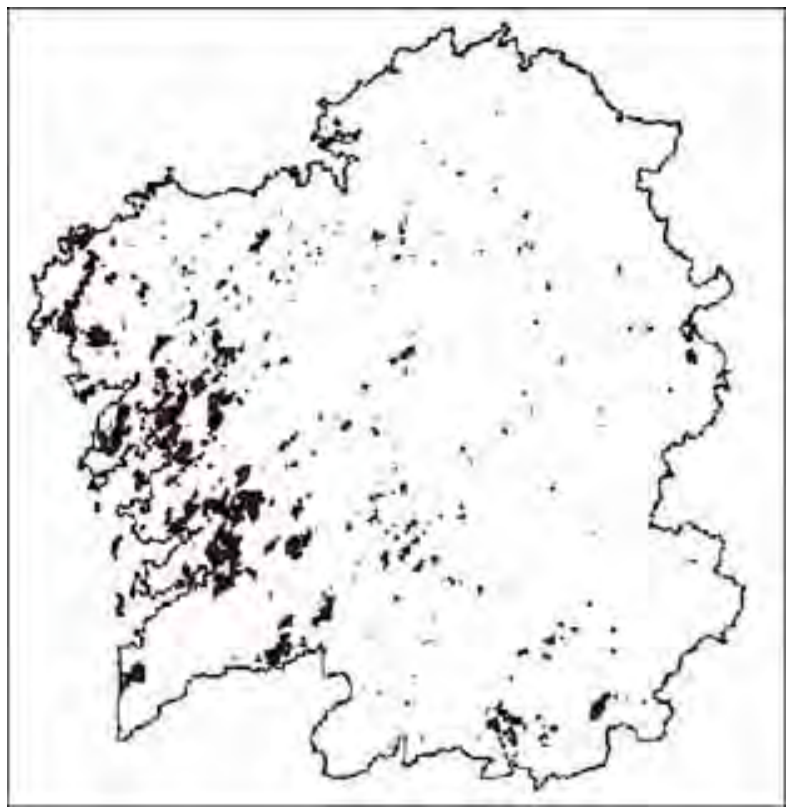

Figura 2. Polígonos con un valor de BAI mayor que 90 (formato vectorial) 


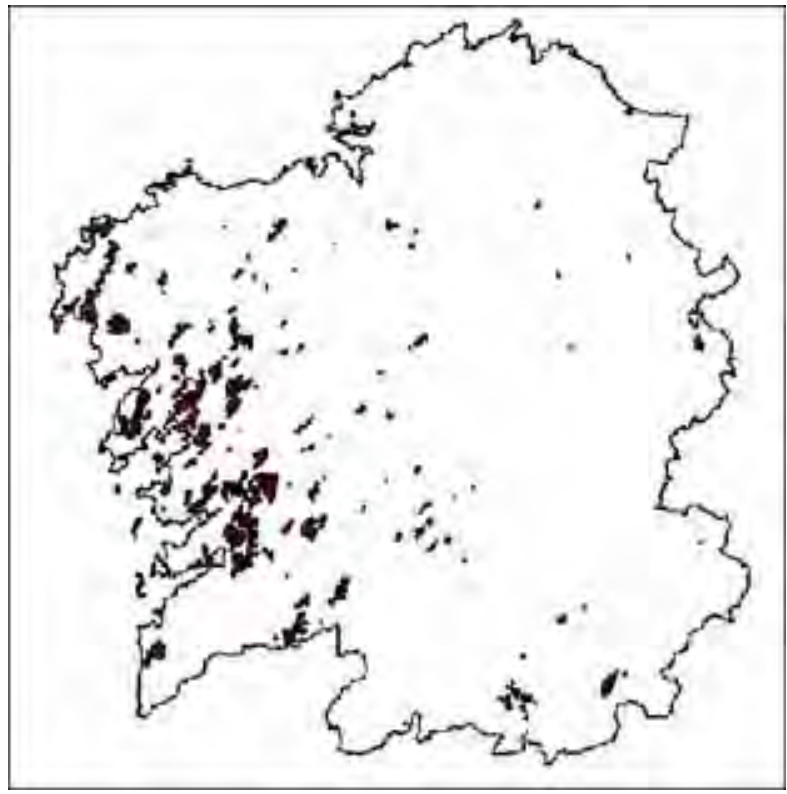

Figura 3. Polígonos con un valor de BAI mayor que 90 y conteniendo al menos un hotspot (formato vectorial)

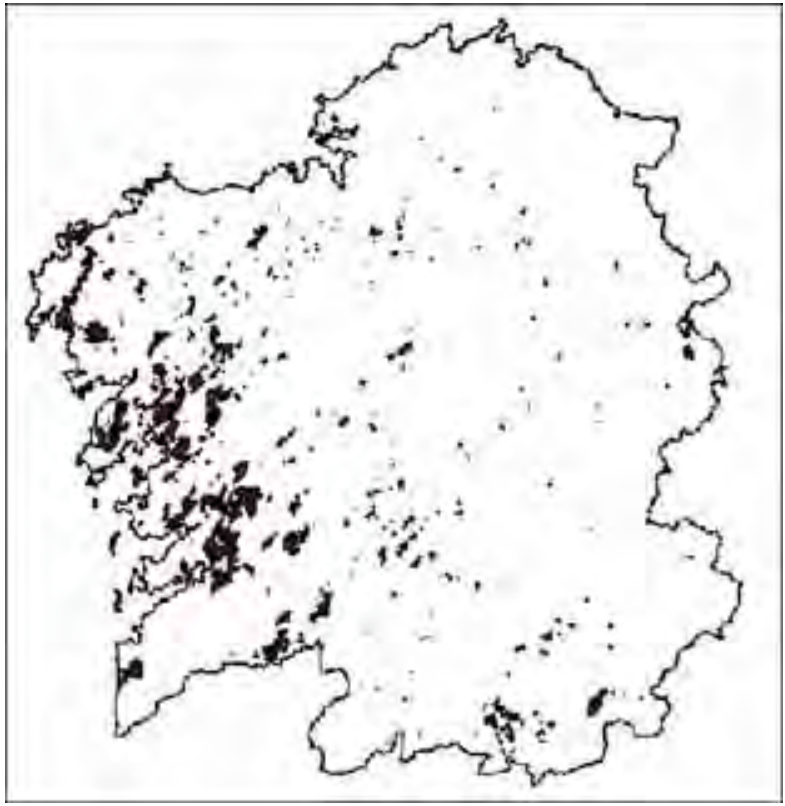

Figura 4. Mapa final de área afectada por el fuego compuesto por: (i) polígonos con un valor de BAI mayor que 90 y conteniendo al menos un hotspot y (ii) polígonos recuperados manualmente 
La estimación y cartografía de áreas quemadas a partir de datos AWiFS y MODIS ha resultado rápida y fiable. El uso del índice de área quemada BAI (calculado a partir de datos $\mathrm{AWiFS}$ ) en combinación con la información relativa a anomalías térmicas (derivada de las bandas térmicas de MODIS) en la generación de la cartografía de las áreas afectadas por el fuego, es una metodología innovadora e interesante por los motivos siguientes: (i) el pre-procesado y procesado de los datos ha sido rápido, (ii) no precisa datos de campo, (iii) apenas necesita de decisiones basadas en la opinión del experto (método objetivo) y (iv) ha resultado de exitosa aplicación a escala regional (Galicia, tres millones de hectáreas).

\section{Agradecimientos}

Los autores desean expresar su agradecimiento al Instituto Geográfico Nacional, por proporcionar la cartografía CORINE Land Cover 2000, así como al MODIS Ground Team de la NASA, por la cesión de los datos MODIS hotspot.

\section{Referencias bibliográficas}

Chuvieco, E., Martín, M. P. y Palacios, A. (2002). Assessment of different spectral indices in the red - near-infrared spectral domain for burned land discrimination. Int. J. Remote Sensing 23 (23): 5103-5110.

González-Alonso, F., Merino-De-Miguel, S., Roldán-Zamarrón, A., García-GigoRRO, S. y CuEvAS, J.M. (2007). MERIS Full Resolution data for mapping level-ofdamage by forest fires: the Valencia de Alcántara event in August 2003. Int. J. Remote Sensing 28 (3-4): 797-809.

Koustias, N., Karteris, M., Fernández-Palacios, A., Navarro, C., Jurado, J., Navarro, R. y Lobo, A. (1999). Burnt land mapping at local scale. En Remote Sensing of Large Wildfires in the European Mediterranean Basin (Chuvieco, E., Ed.). Springer, Berlín, 157-187.

Kulkarni, A. V., Singh, S. K., Mathur, P. y Mishra, V. D. (2006). Algorithm to monitor snow cover using AWiFS data of RESOURCES AT-1 for the Himalayan region. Int. J. Remote Sensing 27 (12): 2449-2457.

Martín, M. P., Gómez, I. y Chuvieco, E. (2005). Performance of a burned-area index (BAIM) for mapping Mediterranean burned scars from MODIS data. En Proceedings of the $5^{\text {th }}$ International Workshop on Remote Sensing and GIS applications to Forest Fire Management: Fire Effects Assessment (De la Riva, J., Pérez-Cabello, F. y Chuvieco, E., Eds.). Universidad de Zaragoza, Zaragoza, 193-197.

Ministerio de Medio Ambiente (2006). Incendios forestales en España 2006. Avance Informativo. Madrid. Disponible en www.incendiosforestales. org.

XunTa de Galicia (2007). Datos sobre superficies quemadas en 2006. Galicia. Disponible en mediorural.xunta.es/forestal/Datos_incendios/ index.php. 